\title{
Off-resonance surface plasmon enhanced spontaneous emission from CdTe quantum dots
}

\author{
Vamsi K. Komarala, ${ }^{\text {a) }}$ Yury P. Rakovich, and A. L. Bradley \\ Semiconductor Photonics Group, School of Physics, Trinity College Dublin, Dublin 2, Ireland
}

Stephen J. Byrne and Yurii K. Gun'ko

School of Chemistry, Trinity College Dublin, Dublin 2, Ireland

N. Gaponik and A. Eychmüller

Institute of Physical Chemistry, University of Hamburg, 20146 Hamburg, Germany

(Received 14 September 2006; accepted 16 November 2006; published online 22 December 2006)

\begin{abstract}
Surface plasmon (SP) enhanced photoluminescence (PL) from CdTe quantum dots (QDs) on monolayers of $\mathrm{Au}$ nanoparticles is investigated under both resonant and nonresonant conditions. Enhancement of the QD PL intensity is observed when the emission spectrum is redshifted with respect to the SP absorption resonance. Coupling to the SPs results in a redshift and broadening of the PL spectrum, and an increase in the PL decay rate. The largest coupling is observed for QD monolayers with peak emission at $667 \mathrm{~nm}$, producing a ten fold increase in PL intensity. No change in PL intensity and decay rate is observed at the SP resonance. (c) 2006 American Institute of Physics. [DOI: 10.1063/1.2422906]
\end{abstract}

Surface plasmons (SPs) excited on thin metallic structures/nanoparticles play a major role in photoluminescence (PL) enhancement ${ }^{1-4}$ and PL quenching. ${ }^{5,6}$ These effects have been attributed to enhanced excitation field, ${ }^{2-5}$ modified radiative and nonradiative decay rates, ${ }^{7-9}$ and coupling to SPs. ${ }^{3,4,7}$ In a metal-emitter system, the exact conditions under which enhancement or quenching of the PL will occur are still under debate. We report on the offresonance enhancement of the quantum dot (QD) emission and the importance of SP scattering.

The structures, grown using a layer-by-layer technique, ${ }^{10,11}$ are comprised of 3 monolayers (ML) of $\mathrm{Au}$ NPs (diameter $\sim 7 \mathrm{~nm}$ ) on a quartz substrate, followed by a polyelectrolyte (PE) spacer layer and capped by $2 \mathrm{ML}$ of CdTe thioglycolic acid stabilized QDs. ${ }^{12-14}$ The $3 \mathrm{ML}$ of Au NPs and 2 ML of QDs on quartz substrates are deposited using positively charged polyethyleneimine as the counterpart. The spacer is comprised of bilayers of positively charged poly(diallyldimethylammonium chloride) and negatively charged poly(sodium 4-styrene sulfonate), with thicknesses of approximately 1.4 and $11.7 \mathrm{~nm}$ for one and nine PE bilayers, respectively. ${ }^{15}$ Room temperature PL spectra are recorded with the excitation wavelength of $400 \mathrm{~nm}$ using a Perkin-Elmer fluorescence spectrometer. The time-resolved PL decays were measured using a PicoQuant Microtime200 time-resolved confocal microscope system with 150 ps resolution. Excitation is provided by $480 \mathrm{~nm}$ picosecond pulses at a $10 \mathrm{MHz}$ repetition rate from an LDH-480 laser head controlled by a PDL-800B driver (PicoQuant).

The PL spectra for CdTe QD monolayers, with peak emissions at 556, 612, 667, and $757 \mathrm{~nm}$, were recorded as a function of QD-metal separation. The PL intensity enhancement factor is determined by a comparison with the PL peak intensity in the absence of the Au NP layer. A tenfold PL peak intensity enhancement was observed for QDs emitting at $667 \mathrm{~nm}$, whereas a fourfold increase was noted for those

\footnotetext{
${ }^{a)}$ Electronic mail: komaralv@tcd.ie
}

emitting at 612 and $757 \mathrm{~nm}$ and essentially no discernible change was observed for the $556 \mathrm{~nm}$ QDs, as shown in Fig. 1 . The PL peak wavelength and the full width at half maximum (FWHM) as a function of the number of PE bilayers, for the 667 and $556 \mathrm{~nm}$ QDs, are shown in Fig. 2 (the data for the 612 and $757 \mathrm{~nm}$ QDs, are not shown for clarity but are discussed below). Redshifts of the PL peak wavelength $(12,20$, and $10 \mathrm{~nm})$ and broadening of the FWHM $(20,25$, and $5 \mathrm{~nm}$ ), in comparison with the PL spectra measure on the quartz substrate, were observed for the 612, 667, and $757 \mathrm{~nm}$ QDs, respectively. In contrast, the peak wavelength of the $556 \mathrm{~nm}$ QDs is only marginally redshifted by $\sim 5 \mathrm{~nm}$ and is essentially independent of spacer thickness.

For colloids it has been predicted that an excited surface plasmon will radiate whenever the scattering cross section of the colloid is dominant compared to the absorption cross

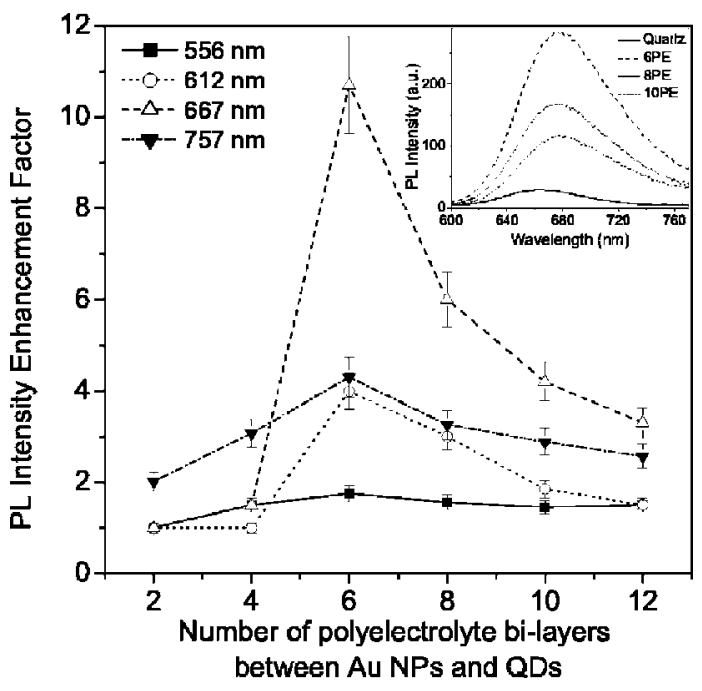

FIG. 1. Dependence of the PL intensity enhancement factor on the number of polyelectrolyte bilayers forming the metal-QD spacer. Inset: PL spectra for $2 \mathrm{ML}$ of QDs on quartz (peak emission at $\sim 667 \mathrm{~nm}$ ) and on the Au NP layer with spacers of six, eight, and ten PE bilayers. 


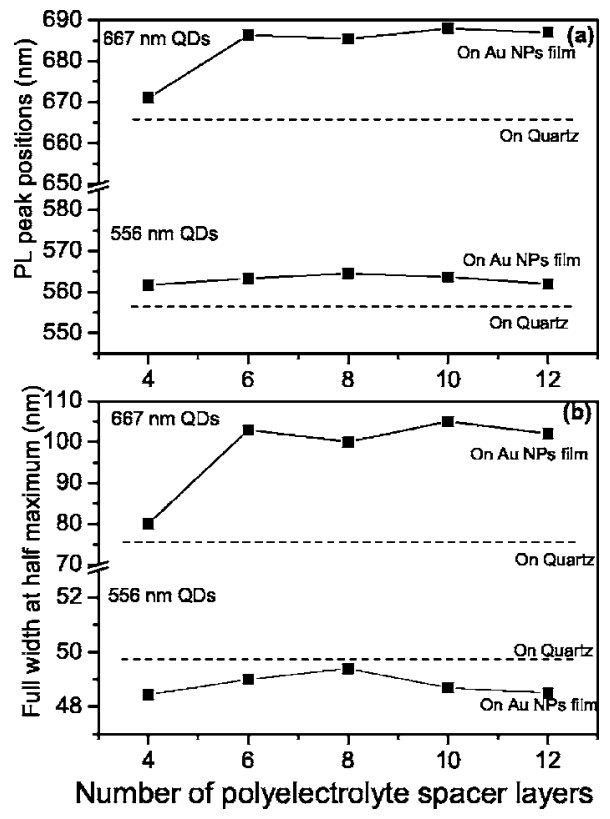

FIG. 2. Comparison of the (a) peak wavelength and (b) full width at half maximum values, with and without the Au NP layer.

section. $^{7} \mathrm{Au}$ NPs of $\sim 7 \mathrm{~nm}$ diameter exhibit negligible scattering and dominant absorption. However, when metal NPs are in close proximity, for example, in arrays and aggregates, there is typically a redshift in the absorption and the introduction of a scattering component at longer wavelengths. ${ }^{16}$ The absorption spectrum for the $3 \mathrm{ML}$ of Au NPs shows a $20 \mathrm{~nm}$ redshift to $\sim 540 \mathrm{~nm}$, indicating that the plasmon properties have been modified and a scattering component can be expected at longer wavelengths.

The observed PL enhancement and spectral modifications are attributed to coupling to the SPs supported by the Au NP layer. The SP is an evanescent wave that decays exponentially with distance from the metal surface. The QD-SP coupling occurs within the evanescent field, leading to distant dependent enhancement. Furthermore, the experimental results indicate that the emission is influenced by the scattering spectrum resulting from the collective excitation of the closely packed NPs. ${ }^{16}$ Consequently, PL enhancement is only expected at wavelengths greater than the SP absorption resonance, where the scattering dominates. This is consistent with our experimental observations. For the $667 \mathrm{~nm}$ QD spectra, shown in the inset of Fig. 1, the longer wavelength spectral components will have a greater overlap with the scattering spectrum and are more strongly enhanced, contributing to the redshift in the peak wavelength and increase in the FWHM. Similar behavior is observed for the $612 \mathrm{~nm}$ QDs, though to a lesser extent indicating a less overlap with the $\mathrm{Au}$ NP layer scattering spectrum. The spectra for the $757 \mathrm{~nm}$ QDs are broadened on the lower wavelength side consistent with the $757 \mathrm{~nm}$ QD emission occurring at longer wavelengths than the peak of the scattering spectrum. Observed redshifts in the PL spectra may also have contributions from enhanced resonant energy transfer between the QDs (Ref. 17) or modified exciton binding energies near the metal surface, ${ }^{18}$ which would account for the small shifts for the 556 and $757 \mathrm{~nm}$ QD emissions.

When the QDs are in direct contact with the Au NP film, the PL is effectively quenched due to the dominance of nonradiative energy transfer to the metal. ${ }^{2}$ Maximum enhance-
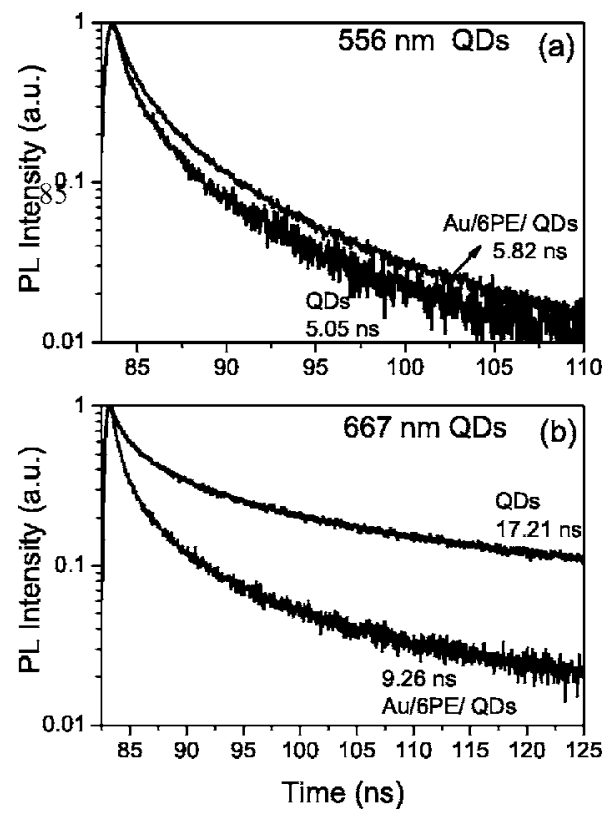

FIG. 3. Comparison of the PL decays for the (a) $556 \mathrm{~nm}$ and (b) $667 \mathrm{~nm}$ emitting QDs, with and without the Au NP layer (including a spacer of six PE bilayers).

ment occurs for a six PE bilayer spacer between QDs and Au NP layer corresponding to $\sim 7.6 \mathrm{~nm}$. As the distance further increases the PL intensity decreases. The enhancement factor is very sensitive to the QD-Au NP layer separation. Similar optimum QD-metal separations have been attributed to the competition between metal enhanced local excitation fields and quenching. ${ }^{19}$ The importance of enhanced excitation was investigated by pumping the $667 \mathrm{~nm}$ QDs at $540 \mathrm{~nm}$, resonant with the Au NP SP absorption band, where a larger local field enhancement can be expected. The PL enhancement factor was unchanged, indicating that enhanced excitation is not a factor in our observations.

The PL decays for the 556 and 667 nm QDs, shown in Fig. 3, exhibit multiexponential behavior which is attributed to band-to-band and surface related emission. Differences in radiative lifetimes for the 556 and $667 \mathrm{~nm}$ QDs are due to the size dependence of the influence of dark exciton states. ${ }^{20}$ The $667 \mathrm{~nm}$ QDs with a six PE bilayer separation from the $\mathrm{Au}$ NP layer show a reduction in the average lifetime. Similar behavior was also observed for the 612 and $757 \mathrm{~nm}$ QDs.

SP-coupled enhancement of the PL intensity has been explained by an additional rate, $\Gamma_{m}$, corresponding to the transfer of energy to the metal, which results in an increase internal quantum efficiency. ${ }^{7,8}$ The enhanced internal quantum yield $\left(Q_{m}\right)$ and PL lifetime $\left(\tau_{m}\right)$ are given by

$$
Q_{m}=\frac{\Gamma+\Gamma_{m}}{\Gamma+\Gamma_{m}+k_{\mathrm{nr}}}
$$

and

$$
\tau_{m}=\frac{1}{\Gamma+\Gamma_{m}+k_{\mathrm{nr}}},
$$

where $\Gamma$ is the radiative rate and $k_{\mathrm{nr}}$ is the nonradiative decay rate. Taking an estimation of the QD monolayer quantum yield of $2 \%$ (Ref. 21) and the measured average lifetimes, $\tau_{0}=17.21 \mathrm{~ns}$ and $\tau_{m}$ of $9.26 \mathrm{~ns}$ for $2 \mathrm{ML}$ of $667 \mathrm{~nm}$ QDs, we obtain $Q_{m} / Q_{0}=23$. This is higher than the experimentally license or copyright; see http://apl.aip.org/about/rights_and_permissions 
observed factor of 10 . However, it is likely that discrepancies are due to competition between PL quenching and PL enhancing processes. ${ }^{5}$ Competing processes would also account for the observation of the maximum PL enhancement at a QD-Au NP separation of six PE bilayers.

The $556 \mathrm{~nm}$ QDs do not show any reduction in lifetime, confirming that they are not efficiently coupled to the SPs, despite being closest to the SP resonance. A number of reports show the strongest QD-SP coupling and maximum PL enhancement for this resonant condition. ${ }^{4,8}$ Many of these studies consider continuous metallic layers where the SP scattering is provided by surface roughness. This is different from our case where the enhancement factor, arising due to the proximity of the Au NPs to each other, has a spectral dependence. For the Au NP layers we do not expect the scattering component to be dominant close to the peak of the SP absorption band. ${ }^{16}$ These observations suggest that the coupling of the emitted excitons to the SPs and modification of the photon mode density are dependent on the strength of the scattered field. Differences in the magnitude and spectral position of the scattering component with respect to the SP absorption in different metallic structures/clusters account for the diversity of experimental observations reported in the literature.

In conclusion we have demonstrated that SP enhanced emission from CdTe QDs on Au NP layers is achieved when the QD peak emission is redshifted with respect to the SP resonance. The experimental results suggest that the spectral overlap with the scattering component determines the magnitude of the enhancement and emission properties of the coupled system. Layers of Au NPs can be used, despite the fact that there is no scattering component for the individual NPs.
The authors thank John F. Donegan for fruitful discussions. This research work is funded by Science Foundation Ireland. N.G. and A.E. would like to acknowledge Eu NoE PHOREMOST.

${ }^{1}$ K. T. Shimizu, W. K. Woo, B. R. Fisher, H. J. Eisler, and M. G. Bawendi, Phys. Rev. Lett. 89, 117401 (2002).

${ }^{2}$ O. Kulakovich, N. Strekal, A. Yaroshevich, S. Maskevich, A. Gaponenko, I. Nabiev, U. Woggon, and M. Artemyev, Nano Lett. 2, 1449 (2002).

${ }^{3}$ J. H. Song, T. Atay, S. Shi, H. Urabe, and A. V. Nurmikko, Nano Lett. 5, 1557 (2005).

${ }^{4}$ J. S. Biteen, N. S. Lewis, H. A. Atwater, H. Mertens, and A. Polman, Appl. Phys. Lett. 88, 131109 (2006).

${ }^{5}$ E. Dulkeith, M. Ringler, T. A. Klar, and J. Feldmann, Nano Lett. 5, 585 (2005).

${ }^{6}$ G. Schneider and G. Decher, Nano Lett. 6, 530 (2006).

${ }^{7}$ J. R. Lakowicz, Anal. Biochem. 337, 171 (2005).

${ }^{8}$ K. Okamoto, S. Vyawahare, and A. Scherer, J. Opt. Soc. Am. B 23, 1674 (2006).

${ }^{9}$ J. Lee, A. Govorov, J. Dulka, and N. Kotov, Nano Lett. 4, 2323 (2004).

${ }^{10}$ G. Decher, Science 277, 1232 (1997).

${ }^{11}$ A. Shavel, N. Gaponik, and A. Eychmüller, Eur. J. Inorg. Chem. 2005, 3613 (2005).

${ }^{12}$ N. Gaponik, D. V. Talapin, A. L. Rogach, K. Hoppe, E. V. Shevchenko, A. Kornowski, A. Eychmüller, and H. J. Weller, J. Phys. Chem. B 106, 7177 (2002).

${ }^{13}$ V. K. Komarala, Y. P. Rakovich, A. L. Bradley, S. J. Byrne, S. A. Corr, and Y. K. Gun'ko, Nanotechnology 17, 4117 (2006).

${ }^{14}$ S. J. Byrne, S. A. Corr, T. Y. Rakovich, Y. K. Gun'ko, Y. P. Rakovich, J. F. Donegan, S. Mitchell, and Y. Volkov, J. Mater. Chem. 16, 2896 (2006).

${ }^{15}$ F. Caruso, Adv. Mater. (Weinheim, Ger.) 13, 11 (2001).

${ }^{16}$ A. A. Lazarides and G. C. Schatz, J. Phys. Chem. B 104, 460 (2000).

${ }^{17}$ J. I. Gersten and A. Nitzan, Chem. Phys. Lett. 104, 31 (1984).

${ }^{18}$ K. Zhao, J. Choi, and Y. Lo, Appl. Phys. Lett. 88, 243104 (2006).

${ }^{19}$ P. Anger, P. Bharadwaj, and L. Novotny, Phys. Rev. Lett. 96, 113002 (2006).

${ }^{20}$ A. F. Van Driel, G. Allan, C. Delerue, P. Lodahl, W. L. Vos, and D. Vanmaekelbergh, Phys. Rev. Lett. 95, 236804 (2005).

${ }^{21}$ T. Franzl, A. Shavel, A. L. Rogach, N. Gaponik, T. A. Klar, A. Eychmüller, and J. Feldmann, Small 1, 392 (2005). 